Rechtsanwältin Gabriele Ostermeier erhielt anschließend das Wort und wies die Aufsichtsrats- und Vorstandsmitglieder unter anderem auf langjährige Abschlussquoten von ca. 50 Prozent Frauen betriebswirtschaftlicher oder juristischer Studiengänge hin, die Frauen für Führungspositionen qualifizierten. Ein Ingenieurstudium sei dazu nicht unbedingt notwendig. Dieter Zetsche hatte zuvor bedauert, dass so wenige Frauen Ingenieurwissenschaften studierten und den Mangel an weiblichen Führungskräften bei Daimler unter anderem mit dem hohen Anteil technisch ausgebildeter Mitarbeiter bei Daimler begründet. Ostermeier stellte abschließend die Frage, ob man bei Daimler die Frauen in Führungspositionen überhaupt haben wolle. Der Vorstandsvorsitzende Zetsche betonte, dass man selbstverständlich die Frauen haben wolle und dass es ihm auch bewusst sei, dass die Tatsache, dass bisher nur Männer in den Führungspositionen säßen, ein Handicap bei der Suche nach geeigneten Frauen sei. Dies solle aber geändert werden. Er zeigte sich ebenfalls gesprächsbereit. Der Aufsichtsratsvorsitzende Bischoff warnte abschließend jedoch noch einmal vor der Quote, die „keinesfalls gut für die Frauen“ sei.

Den folgenden Fragesteller bewog diese Antwort dazu, dies mit „Damen gibt es in großer Schar, bei Daimler nur im Singular“ zu kommentieren.
Der djb plant weitere Aktionen wie diese. Die Hauptversammlungen von Münchener Rück und E.ON wurden bereits von Kolleginnen besucht. Als Fragen und Forderungen bei zukünftigen Hauptversammlungen bieten sich beispielsweise an:

- Wie ist die Repräsentanz von Frauen auf den verschiedenen Führungsebenen?

- Werden die Genderprogramme evaluiert und werden im Geschäftsbericht entsprechende Zahlen veröffentlicht bzw. warum geschieht dies nicht?

- In die Findungskommissionen für den Aufsichtsrat sind Frauen zu platzieren.

- In den Corporate Governance Kodex ist eine Frauenquote aufzunehmen.

Wir fordern unsere Mitglieder dazu auf, beim jetzigen niedrigen Stand der Aktienkurse Aktien der größeren (Dax-)Unternehmen zu kaufen, damit wir nicht nur zu zweit oder dritt bei den Hauptversammlungen auftreten können, sondern in Zukunft möglicherweise auch größere Aktionen mit mehr Frauen durchführen können. Bitte melden Sie sich auch, wenn Sie bereits Aktien besitzen und bereit wären, selbst bei einer Aktion mitzumachen oder einer Kollegin eine Untervollmacht zur Teilnahme zu erteilen.

Das macht Spaß und ist publikumswirksam!

\title{
Justitia ist eine Frau
}

\section{Eröffnung der Ausstellung im Bundesjustizministerium, Berlin, am 13. Mai 2009}

Warum ist Justitia eine Frau? Sind Frauen am Ende die gerechteren Menschen? Antwort auf diese und andere Fragen gibt die Wanderausstellung „Füllhorn, Waage, Schwert - Justitia ist eine Frau". Sie führt durch 23.000 Jahre Gerechtigkeitsgeschichte aus Sicht der Frauen. Dieser frauengeschichtliche Blick eröffnet überraschende Einsichten, neue Fragen und anregende Diskussionen. Die Besucherinnen und Besucher

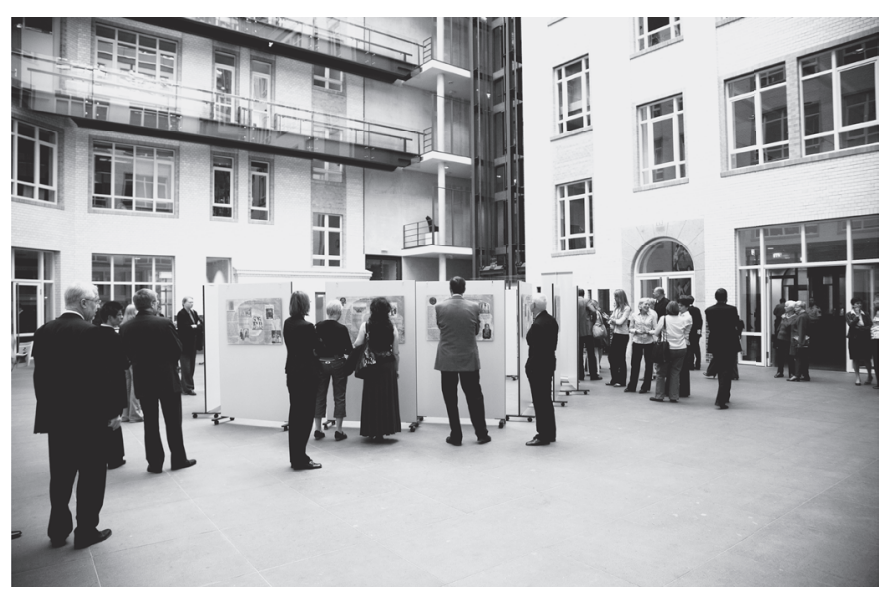

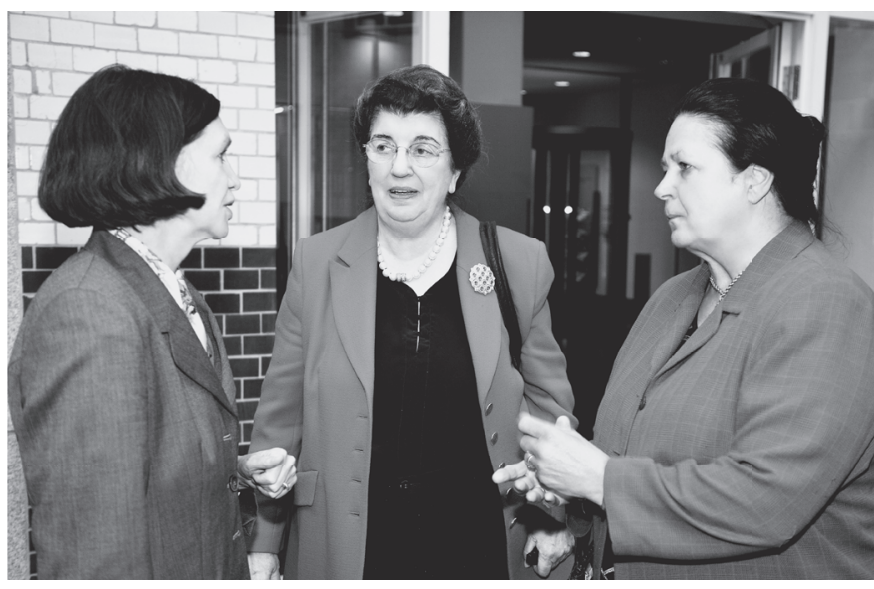

- Namhafte Gäste, v.l.n.r.: die Präsidentin des djb, Rechtsanwältin und Notarin Jutta Wagner, Justizsenatorin a.D. Rechtsanwältin Dr. Lore Maria Peschel-Gutzeit und Justizsenatorin a.D. Rechtsanwältin Karin Schubert.

Eröffnung der Ausstellung im Lichthof des Bundesjustizministeriums, Berlin. 


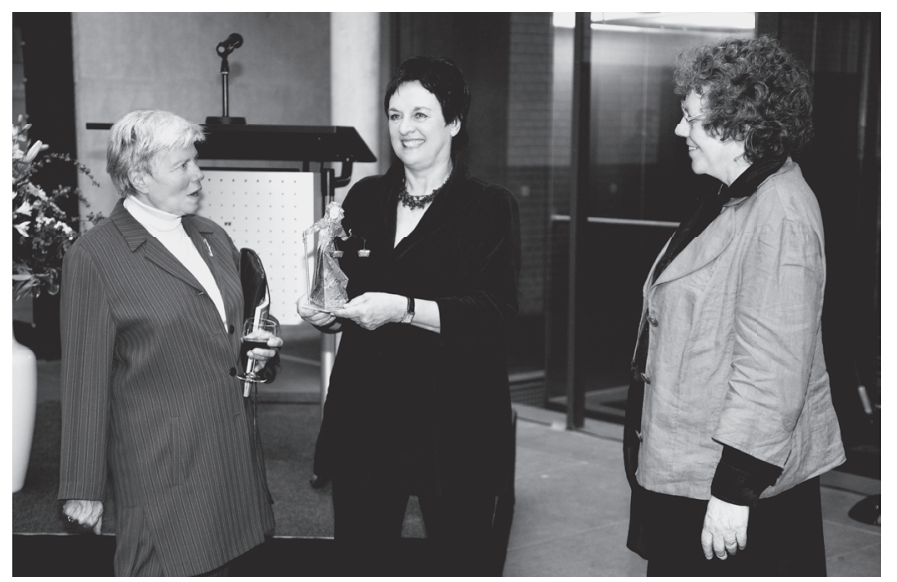

$\Delta$ Nach ihrer Eröffnungsrede überreichte Projektleiterin Dr. Barbara Degen Justizministerin Brigitte Zypries als Gastgeschenk eine Origami-Justitia, ein Unikat der Künstlerin Rita Domgans, Neuss, v.I.n.r.: Wissenschaftliche Leiterin Prof. Dr. Annette Kuhn, Bundesjustizministerin Brigitte Zypries, Projektleiterin Dr. Barbara Degen.

werden angeregt, über ihr eigenes Verhältnis zu Recht, Unrecht und Gerechtigkeit nachzudenken.

Idee und Konzept der Justitia-Ausstellung stammen von Dr. Barbara Degen, Juristin und stellvertretende Vorsitzende des Vereins „Haus der Frauengeschichte e.V.“ (HdFG). Unter der wissenschaftlichen Leitung der Bonner Frauenhistorikerin Prof. Dr. Annette Kuhn, Vorsitzende des HdFG e.V., wirft Barbara Degen auf zwölf Ausstellungstafeln einen eigenen Blick in die Räume der Gerechtigkeit.

Fotos: EVA HeHemann, Düsseldorf

Der djb fördert die Entleihung der Justitia-Ausstellung durch Regionalgruppen und Landesverbände: Nach Beschluss des Bundesvorstands vom 17. November 2007 werden auf Antrag die Entleihgebühr für die Justitia-Ausstellung, die Regionalgruppen und Landesverbänden entstehen, bis zu einer Höhe von 500 EUR je Ausstellung (maximal 1.500,- Euro pro Jahr) übernommen. Der frühzeitig an die Geschäftsstelle zu richtende Antrag muss eine Ausstellungskonzeption und ein Finanzierungskonzept (mit Eigenanteil der Regionalgruppe/des Landesverbandes) enthalten. Als förderungswürdig werden nur solche Ausstellungskonzeptionen angesehen, die einen über den Kreis der Regionalgruppe/des Landesverbandes hinausgehenden „Werbeeffekt" für den djb haben. Über den Antrag entscheidet das Präsidium. Ein Anspruch auf Übernahme der Entleihgebühr besteht nicht.

\section{Die Entwicklung des europäischen Zivilrechts aus der Sicht des Europäischen Gerichtshofs - Was hat der Bürger davon?}

\author{
Prof. Dr. Juliane Kokott, LL.M. (Am. Univ.), S.J.D. (Harvard) \\ Generalanwältin am Gerichtshof der Europäischen Gemein- \\ schaften, Luxemburg
}

Der europäische Gesetzgeber hat die justizielle Zusammenarbeit in Zivilsachen und die Harmonisierung des materiellen Verbraucherrechtes in den letzten Jahren deutlich vorangetrieben. Die Schlagworte lauten: „Brüssel I“, „Brüssel Ila“ sowie „Rom I“ und „Rom II“. Neue gesetzgeberische Initiativen sind auf dem Weg - der Vorschlag für eine Richtlinie über die Rechte der Verbraucher ist nur ein Beispiel. Anlass genug für die Regionalgruppe Brüssel im djb, Frau Prof. Dr. Dr. Juliane Kokott, Generalanwältin am Gerichtshof der Europäischen Gemeinschaften in Luxemburg und selbst djb-Mitglied, für einen Vortrag nach Brüssel einzuladen. Thema: Die Entwicklung des europäischen Zivilrechts aus der Sicht des Europäischen Gerichtshofs - Was hat der Bürger davon?

Die Veranstaltung am 4. Juni 2009 in den Räumen der Bayerischen Landesvertretung war ein voller Erfolg. Die Gastgeberinnen, die bayerische Staatsministerin der Justiz und für Verbrau- cherschutz, Dr. Beate Merk, und Margarete Hofmann, Vorsitzende der Regionalgruppe Brüssel, begrüßten mehr als 250 Gäste aus den EU-Institutionen, internationalen Anwaltskanzleien und Verbänden zu Vortrag und anschließendem Empfang.

Frau Staatsministerin Dr. Beate Merk warnte in ihren einleitenden Worten ${ }^{1}$ davor, die europäische Harmonisierung des Zivilrechts so weit voranzutreiben, dass nationale Verbraucherschutzstandards zurückgenommen werden müssen. Europarechtliche Regelungen seien gerechtfertigt, soweit sie Binnenmarkthindernisse beseitigen. Darüber hinaus dürfe den Mitgliedstaaten ihre Kompetenz im Zivilrecht aber nicht beschnitten werden.

Anschließend stellte Margarete Hofmann als Vorsitzende der RG Brüssel den djb im Allgemeinen und die Aktivitäten der Regionalgruppe Brüssel - einzige Auslandsgruppe des djb - im Besonderen vor. „Klein, aber fein und vor allem sehr rührig“ im einflussreichen djb-Netzwerk - das ist die RG Brüssel!

1 Alle Vorträge sind in voller Länge auf der Webseite des djb zu finden: www.djb.de. 